PROCEEDINGS OF THE

AMERICAN MATHEMATICAL SOCIETY

Volume 140, Number 6, June 2012, Pages 2053-2063

S 0002-9939(2011)11063-3

Article electronically published on October 14, 2011

\title{
TWO CLASSES OF SPECIAL FUNCTIONS USING FOURIER TRANSFORMS OF GENERALIZED ULTRASPHERICAL AND GENERALIZED HERMITE POLYNOMIALS
}

\author{
MOHAMMAD MASJED-JAMEI AND WOLFRAM KOEPF \\ (Communicated by Walter Van Assche)
}

\begin{abstract}
Some orthogonal polynomial systems are mapped onto each other by the Fourier transform. Motivated by the paper [H. T. Koelink, On Jacobi and continuous Hahn polynomials, Proc. Amer. Math. Soc., 124 (1996) 887898], in this paper we introduce two new classes of orthogonal functions, which are respectively Fourier transforms of the generalized ultraspherical polynomials and generalized Hermite polynomials, and then obtain their orthogonality relations using Parseval's identity.
\end{abstract}

\section{INTRODUCTION}

It is known that the generalized ultraspherical (Gegenbauer) polynomials with weight $|x|^{2 a}\left(1-x^{2}\right)^{b}$ on $[-1,1]$ and the generalized Hermite polynomials with weight $|x|^{2 a} e^{-x^{2}}$ on $(-\infty, \infty)$ are two basic examples of sieved orthogonal polynomials [1, 8]. The date of these polynomials goes back to 1984, when Al-Salam, Allaway and Askey [1] observed that some interesting polynomials arise as limiting cases of the continuous $q$-ultraspherical polynomials introduced by L.J. Rogers [16]. They then named them "sieved ultraspherical polynomials of the first and second kind" and showed that the polynomials of the first kind satisfy a three-term recurrence relation as

$$
\begin{cases}C_{n+1}^{\lambda}(x ; k)=2 x C_{n}^{\lambda}(x ; k)-C_{n-1}^{\lambda}(x ; k) & k \neq n, \\ (m+2 \lambda) C_{m k+1}^{\lambda}(x ; k)=2 x(m+\lambda) C_{m k}^{\lambda}(x ; k)-m C_{m k-1}^{\lambda}(x ; k) & m>0\end{cases}
$$

for $C_{0}^{\lambda}(x ; k)=1, C_{1}^{\lambda}(x ; k)=x(k>1)$, while the polynomials of the second kind satisfy the relation

$$
\left\{\begin{aligned}
B_{n+1}^{\lambda}(x ; k)=2 x B_{n}^{\lambda}(x ; k)-B_{n-1}^{\lambda}(x ; k) & k \neq n+1, \\
m B_{m k}^{\lambda}(x ; k)=2 x(m+\lambda) B_{m k-1}^{\lambda}(x ; k) & \\
-(m+2 \lambda) B_{m k-2}^{\lambda}(x ; k) & m>0,
\end{aligned}\right.
$$

in which $B_{0}^{\lambda}(x ; k)=1, B_{1}^{\lambda}(x ; k)=2 x(k>1)$.

Received by the editors August 17, 2010 and, in revised form, January 10, 2011 and February 10, 2011.

2010 Mathematics Subject Classification. Primary 33C45, 42A38.

This research was supported in part by a grant from IPM, No. 89330021, and by a grant from "Bonyade Mellie Nokhbegan", No. PM/1184. 
Al-Salam, Allaway and Askey also derived orthogonality relations of $C_{n}^{\lambda}(x ; k)$ and $B_{n}^{\lambda}(x ; k)$ from the orthogonality relation of the continuous $q$-ultraspherical polynomials and showed that the first and second kind of sieved ultraspherical polynomials are respectively orthogonal on $[-1,1]$ with respect to the weight functions

$$
\begin{aligned}
& W_{1}(x, k, \alpha)=\left(1-x^{2}\right)^{\alpha-1 / 2}\left|U_{k-1}(x)\right|^{2 \alpha}, \\
& W_{2}(x, k, \alpha)=\left(1-x^{2}\right)^{\alpha+1 / 2}\left|U_{k-1}(x)\right|^{2 \alpha},
\end{aligned}
$$

where $k \in \mathbf{N}$ is a fixed integer and $U_{k}(x)$ denotes the second kind of Chebyshev polynomials [5, 9].

Later on, Askey in 3 generalized the above-mentioned results and introduced two further classes of sieved polynomials as limiting cases of the $q$-Wilson polynomials that are respectively orthogonal on $[-1,1]$ with respect to the weight functions

$$
W_{1}(x, k, \alpha, \lambda)=\left(1-x^{2}\right)^{\alpha-1 / 2}\left|U_{k-1}(x)\right|^{2 \alpha}\left|T_{k}(x)\right|^{\lambda}
$$

and

$$
W_{2}(x, k, \alpha, \lambda)=\left(1-x^{2}\right)^{\alpha+1 / 2}\left|U_{k-1}(x)\right|^{2 \alpha}\left|T_{k}(x)\right|^{\lambda},
$$

where $T_{k}(x)$ denotes the first kind of Chebyshev polynomials 9 , 17.

In this paper, we deal with the special case $W_{1}(x, 1, b+1 / 2,2 \alpha)=|x|^{2 \alpha}\left(1-x^{2}\right)^{b}$ on $[-1,1]$ corresponding to the well-known generalized ultraspherical polynomials (GUP), which were first investigated by Chihara in detail [5. Chihara could obtain the general properties of GUP via a direct relation between them and Jacobi orthogonal polynomials [9].

By referring to [14, GUP has the explicit hypergeometric representation

$$
U_{n}^{(a, b)}(x)=x^{n}{ }_{2} F_{1}\left(\begin{array}{c|c}
-[n / 2],-a+1 / 2-[(n+1) / 2] & 1 \\
-a-b-n+1 / 2 & \frac{1}{x^{2}}
\end{array}\right),
$$

where ${ }_{2} F_{1}(\cdot)$ is a special case of the generalized hypergeometric function [4, 11] of order $(p, q)$ as

$$
{ }_{p} F_{q}\left(\begin{array}{c}
a_{1} a_{2} \ldots a_{p} \\
b_{1} b_{2} \ldots b_{q}
\end{array} \mid x\right)=\sum_{k=0}^{\infty} \frac{\left(a_{1}\right)_{k}\left(a_{2}\right)_{k} \ldots\left(a_{p}\right)_{k}}{\left(b_{1}\right)_{k}\left(b_{2}\right)_{k} \ldots\left(b_{q}\right)_{k}} \frac{x^{k}}{k !},
$$

and $(r)_{k}=\prod_{i=0}^{k-1}(r+i)$ denotes the Pochhammer symbol in (1.8). Also, the orthogonality relation of the polynomials (1.7) is explicitly in the form [14]

$$
\begin{aligned}
\int_{-1}^{1}|x|^{2 a}\left(1-x^{2}\right)^{b} U_{n}^{(a, b)}(x) U_{m}^{(a, b)}(x) \mathrm{d} x \\
=\left(\frac{\Gamma(a+1 / 2) \Gamma(b+1)}{\Gamma(a+b+3 / 2)} \prod_{j=1}^{n} \frac{\left(j+\left(1-(-1)^{j}\right) a\right)\left(j+\left(1-(-1)^{j}\right) a+2 b\right)}{(2 j+2 a+2 b-1)(2 j+2 a+2 b+1)}\right) \delta_{n, m} \\
=\Gamma\left(a+\frac{1}{2}\right) \Gamma\left(a+b-\frac{1}{2}\right) \Gamma\left(\left[\frac{n}{2}\right]+1\right) \Gamma\left(b+\frac{1}{2}+\left[\frac{n}{2}\right]\right) \\
\quad \times \frac{\Gamma\left(a+1+\left[\frac{n}{2}\right]+\frac{1-(-1)^{n}}{2}\right) \Gamma\left(a+b+\frac{1}{2}+\left[\frac{n}{2}\right]+\frac{1-(-1)^{n}}{2}\right)}{\Gamma(a+1) \Gamma\left(a+b+\frac{3}{2}\right) \Gamma\left(a+b+n-\frac{1}{2}\right) \Gamma\left(a+b+n+\frac{1}{2}\right)} \delta_{n, m},
\end{aligned}
$$


provided that $a+1 / 2>0, a+b-1 / 2>0, \delta_{n, m}=\left\{\begin{array}{ll}0 & (n \neq m), \\ 1 & (n=m),\end{array}\right.$ and $\Gamma(z)=$ $\int_{0}^{\infty} x^{z-1} e^{-x} \mathrm{~d} x(\operatorname{Re}(z)>0)$ denotes the well-known Gamma function satisfying the fundamental recurrence relation $\Gamma(z+1)=z \Gamma(z)$.

Similarly, the generalized Hermite polynomials (GHP) were first introduced by Szegö in [17] giving an explicit second order differential equation. These polynomials can be characterized by using a direct relationship between them and Laguerre orthogonal polynomials 9 .

By referring to [14, the hypergeometric representation of GHP is explicitly in the form

$$
H_{n}^{(a)}(x)=x_{2}^{n} F_{0}\left(\begin{array}{c|c}
-[n / 2],-[n / 2]-a+(-1)^{n} / 2 & -\frac{1}{x^{2}}
\end{array}\right) .
$$

Moreover, the orthogonality relation of these polynomials takes the form

$$
\begin{aligned}
\int_{-\infty}^{\infty} & |x|^{2 a} e^{-x^{2}} H_{n}^{(a)}(x) H_{m}^{(a)}(x) \mathrm{d} x \\
& =\left(\frac{1}{2^{n}} \prod_{i=1}^{n}\left(1-(-1)^{i}\right) a+i\right) \Gamma\left(a+\frac{1}{2}\right) \delta_{n, m} \\
& =\Gamma\left(a+\frac{1}{2}+\left[\frac{n+1}{2}\right]\right) \Gamma\left(\left[\frac{n}{2}\right]+1\right) \delta_{n, m} \quad(a>-1 / 2) .
\end{aligned}
$$

But, it is known that some orthogonal polynomial systems are mapped onto each other by the Fourier transform or other integral transforms such as the Mellin and Hankel transforms 77. The best-known examples of this type are the Hermite functions, i.e. the Hermite polynomials $H_{n}(x)$ multiplied by $\exp \left(-x^{2} / 2\right)$, which are eigenfunctions of the Fourier transform; see also [12, 13, 15] in this regard.

Especially in [10, Koelink showed that the Jacobi and continuous Hahn polynomials can be mapped onto each other in such a way, and the orthogonality relations for the continuous Hahn polynomials then follow from the orthogonality relations of the Jacobi polynomials and using the Parseval identity. Motivated by Koelink's paper, in this section we apply this approach for GUP and GHP to introduce two new classes of orthogonal functions by using Fourier transforms and the Parseval identity.

\section{Fourier transForms of GUP AND GHP AND THEIR ORTHOGONALITY RELATIONS}

To derive the Fourier transforms of GUP and GHP defined in (1.7) and (1.10), we will need the Beta integral having various definitions as

$$
\begin{aligned}
B\left(\lambda_{1} ; \lambda_{2}\right) & =\int_{0}^{1} x^{\lambda_{1}-1}(1-x)^{\lambda_{2}-1} \mathrm{~d} x=\int_{-1}^{1} x^{2 \lambda_{1}-1}\left(1-x^{2}\right)^{\lambda_{2}-1} \mathrm{~d} x \\
& =\int_{0}^{\infty} \frac{x^{\lambda_{1}-1}}{(1+x)^{\lambda_{1}+\lambda_{2}}} \mathrm{~d} x=2 \int_{0}^{\pi / 2} \sin ^{\left(2 \lambda_{1}-1\right)} x \cos ^{\left(2 \lambda_{2}-1\right)} x \mathrm{~d} x \\
& =\frac{\Gamma\left(\lambda_{1}\right) \Gamma\left(\lambda_{2}\right)}{\Gamma\left(\lambda_{1}+\lambda_{2}\right)}=B\left(\lambda_{2} ; \lambda_{1}\right) .
\end{aligned}
$$


The Fourier transform of a function, say $g(x)$, is defined as [7]

$$
\mathbf{F}(s)=\mathbf{F}(g)(s)=\int_{-\infty}^{\infty} e^{-i s x} g(x) \mathrm{d} x,
$$

and for the inverse transform one has the formula

$$
g(x)=\frac{1}{2 \pi} \int_{-\infty}^{\infty} e^{i s x} \mathbf{F}(s) \mathrm{d} s .
$$

For $g, h \in L^{2}(\mathbb{R})$, the Parseval identity related to Fourier theory is given by [7]:

$$
\int_{-\infty}^{\infty} g(x) \overline{h(x)} \mathrm{d} x=\frac{1}{2 \pi} \int_{-\infty}^{\infty} \mathbf{F}(g)(s) \overline{\mathbf{F}(h)(s)} \mathrm{d} s .
$$

By noting (1.9) and (2.4) and the fact that $|x|^{2 a}=x^{2 a} \Leftrightarrow(-1)^{2 a}=1$, we now define the following specific functions:

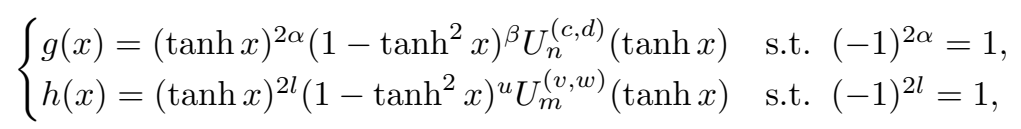

in terms of GUP, to which we will apply the Fourier transform. Clearly for both of the above functions the Fourier transform exists. For instance, for the function $g(x)$ defined in (2.5) we get

$$
\begin{aligned}
\mathbf{F}(g)(s)= & \int_{-\infty}^{\infty} e^{-i s x}(\tanh x)^{2 \alpha}\left(1-\tanh ^{2} x\right)^{\beta} U_{n}^{(c, d)}(\tanh x) \mathrm{d} x \\
= & \int_{-1}^{1}(1+t)^{-\frac{i s}{2}}(1-t)^{\frac{i s}{2}} t^{2 \alpha}\left(1-t^{2}\right)^{\beta-1} U_{n}^{(c, d)}(t) \mathrm{d} t \\
= & 2^{2 \beta-1} \int_{0}^{1}(1-z)^{-\frac{i s}{2}} z^{\frac{i s}{2}}(1-2 z)^{2 \alpha} z^{\beta-1}(1-z)^{\beta-1} U_{n}^{(c, d)}(1-2 z) \mathrm{d} z \\
= & 2^{2 \beta-1} \int_{0}^{1}(1-z)^{\beta-1-\frac{i s}{2}} z^{\beta-1+\frac{i s}{2}}(1-2 z)^{2 \alpha+n} \\
& \quad \times\left(\sum_{k=0}^{[n / 2]} \frac{\left(-\left[\frac{n}{2}\right]\right)_{k}\left(\frac{1}{2}-c-\left[\frac{n+1}{2}\right]\right)_{k}}{(-c-d-n+1 / 2)_{k} k !} \cdot \frac{1}{(1-2 z)^{2 k}}\right) \mathrm{d} z \\
= & 2^{2 \beta-1} \sum_{k=0}^{[n / 2]} \frac{\left(-\left[\frac{n}{2}\right]\right)_{k}\left(\frac{1}{2}-c-\left[\frac{n+1}{2}\right]\right)_{k}}{(-c-d-n+1 / 2)_{k} k !} \\
& \times\left(\int_{0}^{1}(1-z)^{\beta-1-\frac{i s}{2}} z^{\beta-1+\frac{i s}{2}}(1-2 z)^{2 \alpha+n-2 k} \mathrm{~d} z\right) .
\end{aligned}
$$

On the other hand, since

$$
{ }_{2} F_{1}\left(\begin{array}{c|c}
a, b & x \\
c & x
\end{array}\right)=\frac{\Gamma(c)}{\Gamma(b) \Gamma(c-b)} \int_{0}^{1}(1-z)^{c-b-1} z^{b-1}(1-x z)^{-a} \mathrm{~d} z
$$

is the integral representation of the Gauss hypergeometric function [4, 11] for $\operatorname{Re} c>$ $\operatorname{Re} b>0$ and $|x| \leq 1$, the last integral in (2.6) can be shown in terms of (2.7), and 
we finally obtain

$$
\begin{aligned}
\mathbf{F}(g)(s)=2^{2 \beta-1} B\left(\beta+\frac{i s}{2}, \beta-\frac{i s}{2}\right) \sum_{k=0}^{[n / 2]} \frac{\left(-\left[\frac{n}{2}\right]\right)_{k}\left(\frac{1}{2}-c-\left[\frac{n+1}{2}\right]\right)_{k}}{(-c-d-n+1 / 2)_{k} k !} \\
\quad \times{ }_{2} F_{1}\left(\begin{array}{c}
2 k-n-2 \alpha, \beta+\frac{i s}{2} \\
2 \beta
\end{array} \mid 2\right) .
\end{aligned}
$$

Remark 2.1. Since the series ${ }_{2} F_{1}(\cdot)$ in (2.8) does not generally terminate and also $2 \notin[-1,1]$, the evaluation at $x=2$ is rather troublesome unless we assume in (2.6) or (2.8) that $\operatorname{Re}(\beta)>0$ and $\operatorname{Re}(2 \alpha+n-2 k)>-1$.

For simplicity if we define

$$
\begin{aligned}
& K_{n}\left(x ; p_{1}, p_{2}, p_{3}, p_{4}\right) \\
& \quad=\sum_{k=0}^{[n / 2]} \frac{\left(-\left[\frac{n}{2}\right]\right)_{k}\left(\frac{1}{2}-p_{3}-\left[\frac{n+1}{2}\right]\right)_{k}}{\left(-p_{3}-p_{4}-n+1 / 2\right)_{k} k !} F_{1}\left(\begin{array}{c|c}
2 k-n-2 p_{1}, p_{2}+\frac{x}{2} & 2 \\
2 p_{2} &
\end{array}\right),
\end{aligned}
$$

then it is clear in (2.8) that

$$
\mathbf{F}(g)(s)=\frac{2^{2 \beta}-1}{\Gamma(2 \beta)} \Gamma\left(\beta+\frac{i s}{2}\right) \Gamma\left(\beta-\frac{i s}{2}\right) K_{n}(i s ; \alpha, \beta, c, d) .
$$

Now, by substituting (2.10) in Parseval's identity (2.4) and noting (2.5), we get

$$
\begin{aligned}
& 2 \pi \int_{-\infty}^{\infty}(\tanh x)^{2(\alpha+l)}\left(1-\tanh ^{2} x\right)^{\beta+u} U_{n}^{(c, d)}(\tanh x) U_{m}^{(v, w)}(\tanh x) \mathrm{d} x \\
&=2 \pi \int_{-1}^{1} t^{2(\alpha+l)}\left(1-t^{2}\right)^{\beta+u-1} U_{n}^{(c, d)}(t) U_{m}^{(v, w)}(t) \mathrm{d} t \\
&= \frac{2^{2 \beta+2 u-2}}{\Gamma(2 \beta) \Gamma(2 u)} \int_{-\infty}^{\infty} \Gamma\left(\beta+\frac{i s}{2}\right) \Gamma\left(\beta-\frac{i s}{2}\right) \overline{\Gamma\left(u+\frac{i s}{2}\right) \Gamma\left(u-\frac{i s}{2}\right)} \\
& \quad \times K_{n}(i s ; a, b, c, d) \overline{K_{m}(i s ; l, u, v, w)} \mathrm{d} s
\end{aligned}
$$

On the other hand, if in the left-hand side of (2.11) we take

$$
c=v=\alpha+l \text { and } \quad d=w=\beta+u-1,
$$

then according to the orthogonality relation (1.9), equation (2.11) finally reads as

$$
\begin{aligned}
& \frac{1}{2 \pi} \int_{-\infty}^{\infty} \Gamma\left(\beta+\frac{i s}{2}\right) \Gamma\left(\beta-\frac{i s}{2}\right) \Gamma\left(u+\frac{i s}{2}\right) \Gamma\left(u-\frac{i s}{2}\right) \\
& \quad \times K_{n}(i s ; \alpha, \beta, \alpha+l, \beta+u-1) K_{m}(-i s ; l, u, \alpha+l, \beta+u-1) \mathrm{d} s \\
& =\frac{\Gamma(2 \beta) \Gamma(2 u) \Gamma\left(\alpha+l+\frac{1}{2}\right) \Gamma(\beta+u)}{2^{2 \beta+2 u+2 n-2} \Gamma\left(\alpha+\beta+l+u+\frac{1}{2}\right)} \\
& \quad \times \prod_{j=1}^{n} \frac{\left(j+\left(1-(-1)^{j}\right)(\alpha+l)\right)\left(j+\left(1-(-1)^{j}\right)(\alpha+l)+2 \beta+2 u-1\right)}{\left(j+\alpha+\beta+l+u-\frac{3}{2}\right)\left(j+\alpha+\beta+l+u-\frac{1}{2}\right)} \delta_{n, m},
\end{aligned}
$$

where $\prod_{j=1}^{n}(\cdot)=1, \beta, u>0$, and $\alpha+l>-\frac{1}{2}$. 
Remark 2.2. Note that $K_{n}\left(x ; p_{1}, p_{2}, p_{3}, p_{4}\right)$ in (2.9) is not in general a polynomial function and it is reduced to a polynomial of degree $n$ only when $p_{1}=0$. This may be a reason why we could not derive an analogue or a sieved type of continuous Hahn polynomials in relations (2.6) to (2.13).

Remark 2.3. Replacing $n=m=0$ and $\alpha=l=0$ in (2.13) gives a special case of the Barnes beta integral [18,

$$
\begin{array}{r}
\frac{1}{2 \pi} \int_{-\infty}^{\infty} \Gamma(a+i x) \Gamma(b+i x) \Gamma(c-i x) \Gamma(d-i x) \mathrm{d} x \\
=\frac{\Gamma(a+d) \Gamma(a+c) \Gamma(b+d) \Gamma(b+c)}{\Gamma(a+b+c+d)},
\end{array}
$$

if one applies the duplication Legendre formula [ 6 ]

$$
\Gamma(2 z)=\frac{2^{2 z-1}}{\sqrt{\pi}} \Gamma(z) \Gamma\left(z+\frac{1}{2}\right) .
$$

Moreover, the weight function corresponding to the orthogonality relation (2.13) can be simplified by using the Cauchy beta integral $[2,6$,

$$
\frac{1}{2 \pi} \int_{-\infty}^{\infty} \frac{\mathrm{d} t}{(a+i t)^{c}(b-i t)^{d}}=\frac{\Gamma(c+d-1)}{\Gamma(c) \Gamma(d)}(a+b)^{1-(c+d)}
$$

and one of its consequences, i.e.

$$
\Gamma(p+i q) \Gamma(p-i q)=\frac{2^{2-2 p} \pi \Gamma(2 p-1)}{\int_{-\frac{\pi}{2}}^{\frac{\pi}{2}} e^{2 q x} \cos ^{2 p-2} x \mathrm{~d} x},
$$

which results in

$$
\begin{aligned}
\Gamma\left(\beta+\frac{i s}{2}\right) \Gamma\left(\beta-\frac{i s}{2}\right) \Gamma\left(u+\frac{i s}{2}\right) \Gamma\left(u-\frac{i s}{2}\right) \\
=\frac{2^{4-2 \beta-2 u} \pi^{2} \Gamma(2 \beta-1) \Gamma(2 u-1)}{\int_{-\frac{\pi}{2}}^{\frac{\pi}{2}} e^{s x} \cos ^{2 \beta-2} x \mathrm{~d} x \int_{-\frac{\pi}{2}}^{\frac{\pi}{2}} e^{s x} \cos ^{2 u-2} x \mathrm{~d} x} .
\end{aligned}
$$

Therefore, if we define

$$
W^{*}(x ; \lambda)=\int_{-\frac{\pi}{2}}^{\frac{\pi}{2}} e^{x \theta} \cos ^{2 \lambda-2} \theta \mathrm{d} \theta
$$

then the following theorem will eventually be derived.

Theorem 2.4. The special function $K_{n}\left(x ; p_{1}, p_{2}, p_{3}, p_{4}\right)$ defined in (2.9) satisfies an orthogonality relation as

$$
\begin{aligned}
\int_{-\infty}^{\infty} & \frac{K_{n}(i x ; \alpha, \beta, \alpha+l, \beta+u-1) K_{m}(-i x ; l, u, \alpha+l, \beta+u-1)}{W^{*}(x ; \beta) W^{*}(x ; u)} \mathrm{d} x \\
= & \frac{(2 u-1)(2 \beta-1)}{\pi 2^{2 n+1}} \times \frac{\Gamma\left(\alpha+l+\frac{1}{2}\right) \Gamma(\beta+u)}{\Gamma\left(\alpha+\beta+l+u+\frac{1}{2}\right)} \\
& \times \prod_{j=1}^{n} \frac{\left(j+\left(1-(-1)^{j}\right)(\alpha+l)\right)\left(j+\left(1-(-1)^{j}\right)(\alpha+l)+2 \beta+2 u-2\right)}{\left(j+\alpha+\beta+l+u-\frac{3}{2}\right)\left(j+\alpha+\beta+l+u-\frac{1}{2}\right)} \delta_{n, m},
\end{aligned}
$$

where $\beta, u>\frac{1}{2}, \alpha+l>-\frac{1}{2}$ and $W^{*}(x ; \cdot)$ is defined as (2.19). 
For instance, for $\beta=u=1$ the relation (2.20) is reduced to

$$
\begin{aligned}
\int_{-\infty}^{\infty} & \frac{x^{2}}{\sinh ^{2}\left(\frac{\pi x}{2}\right)} K_{n}(i x ; \alpha, 1, r, 1) K_{m}(-i x ; r-\alpha, 1, r, 1) \mathrm{d} x \\
= & \frac{\Gamma\left(r+\frac{1}{2}\right) \Gamma\left(r+\frac{2}{3}\right)}{\pi 2^{2 n-1} \Gamma\left(r+\frac{3}{2}+n\right) \Gamma\left(r+\frac{5}{2}+n\right)} \\
& \times \prod_{j=1}^{n}\left(j+\left(1-(-1)^{j}\right) r\right)\left(j+\left(1-(-1)^{j}\right) r+2\right) \delta_{n, m},
\end{aligned}
$$

where $r=\alpha+l>-\frac{1}{2}$.

The mentioned approach can similarly be applied to the generalized Hermite polynomials. For this purpose, we first define the following specific functions:

$$
u(x)=x^{2 a} e^{-\frac{1}{2} x^{2}} H_{n}^{(b)}(x) \text { and } v(x)=x^{2 c} e^{-\frac{1}{2} x^{2}} H_{m}^{(d)}(x),
$$

for $(-1)^{2 a}=(-1)^{2 c}=1$.

If we take the Fourier transform for e.g. $u(x)$, then we have

$$
\begin{aligned}
\mathbf{F}(u)(s)= & \int_{-\infty}^{\infty} e^{-i s x} x^{2 a} e^{-\frac{1}{2} x^{2}} H_{n}^{(b)}(x) \mathrm{d} x \\
= & \int_{-\infty}^{\infty} e^{-i s x} e^{-\frac{1}{2} x^{2}} x^{2 a+n} \\
& \quad \times\left(\sum_{k=0}^{\left[\frac{n}{2}\right]} \frac{\left(-\left[\frac{n}{2}\right]\right)_{k}\left(-\left[\frac{n}{2}\right]-b+\frac{(-1)^{n}}{2}\right)_{k}}{k !}\left(-x^{-2}\right)^{k}\right) \mathrm{d} x \\
= & \sum_{k=0}^{\left[\frac{n}{2}\right]} \frac{\left(-\left[\frac{n}{2}\right]\right)_{k}\left(-\left[\frac{n}{2}\right]-b+\frac{(-1)^{n}}{2}\right)_{k}}{k !}(-1)^{k} \\
& \times\left(\int_{-\infty}^{\infty} e^{-i s x} e^{-\frac{1}{2} x^{2}} x^{2 a+n-2 k} \mathrm{~d} x\right) .
\end{aligned}
$$

So, it remains in (2.23) to evaluate the definite integral:

$$
I_{n, k}(s ; a)=\int_{-\infty}^{\infty} e^{-i s x} e^{-\frac{1}{2} x^{2}} x^{2 a+n-2 k} \mathrm{~d} x .
$$

There are two ways to compute the above integral. First, by noting that $(-1)^{2 a}=$ 1 in (2.24), we can directly compute $I_{n, k}(s ; a)$ for $n=2 m$ as follows:

$$
\begin{aligned}
I_{2 m, k}(s ; a) & =\int_{-\infty}^{\infty}\left(\sum_{j=0}^{\infty} \frac{(-i s x)^{j}}{j !}\right) x^{2 a+2 m-2 k} e^{-\frac{1}{2} x^{2}} \mathrm{~d} x \\
& =\sum_{j=0}^{\infty} \frac{(-1)^{j} i^{j} s^{j}}{j !}\left(\int_{-\infty}^{\infty} x^{j+2 a+2 m-2 k} e^{-\frac{1}{2} x^{2}} \mathrm{~d} x\right) \\
& =\sum_{r=0}^{\infty} \frac{(-1)^{r} s^{2 r}}{(2 r) !}\left(2 \int_{0}^{\infty} x^{2 r+2 a+2 m-2 k} e^{-\frac{1}{2} x^{2}} \mathrm{~d} x\right) \\
& =\sum_{r=0}^{\infty} \frac{(-1)^{r} s^{2 r}}{(2 r) !} 2^{r+a+m-k+\frac{1}{2}} \Gamma\left(r+a+m-k+\frac{1}{2}\right) .
\end{aligned}
$$


To simplify the last summation of (2.25) in terms of a hypergeometric form we need to use (2.16) and the relations

$$
\Gamma(a+k)=(a)_{k} \Gamma(a), \quad \Gamma(a-k)=\frac{(-1)^{k} \Gamma(a)}{(1-a)_{k}}, \quad \text { and } \quad(2 n) !=\left(\frac{1}{2}\right)_{n} 2^{2 n} n !
$$

Therefore, 2.25) becomes

$$
\begin{aligned}
I_{2 m, k}(s ; a) & =\sum_{r=0}^{\infty} \frac{(-1)^{r} s^{2 r} 2^{r+a+m-k+\frac{1}{2}} \Gamma\left(a+m-k+\frac{1}{2}\right)\left(a+m-k+\frac{1}{2}\right)_{r}}{\left(\frac{1}{2}\right)_{r} 2^{2 r} r !} \\
& =2^{a+m-k+\frac{1}{2}} \Gamma\left(a+m-k+\frac{1}{2}\right)_{1} F_{1}\left(\begin{array}{c}
a+m-k+\frac{1}{2} \\
\frac{1}{2}
\end{array} \mid-\frac{s^{2}}{2}\right) .
\end{aligned}
$$

This computational method can similarly be applied for $I_{2 m+1, k}(s ; a)$. We should just note in this turn that $\int_{-\infty}^{\infty} x^{j+2 a+2 m+1-2 k} e^{\frac{-x^{2}}{2}} \mathrm{~d} x=0$ for any $j=0,2,4, \ldots$ After some computations we obtain

$$
I_{2 m+1, k}(s ; a)=(-i s) 2^{a+m-k+\frac{3}{2}} \Gamma\left(a+m-k+\frac{3}{2}\right)_{1} F_{1}\left(\begin{array}{c}
a+m-k+\frac{3}{2} \\
\frac{3}{2}
\end{array} \mid-\frac{s^{2}}{2}\right) .
$$

Thus, by combining both relations (2.27) and (2.28) and using the well-known identity $\left[\frac{n+1}{2}\right]-\left[\frac{n}{2}\right]=\frac{1-(-1)^{n}}{2}$ we finally have

$$
\begin{aligned}
I_{n, k}(s ; a)=2^{a-k+\frac{1}{2}+\left[\frac{n+1}{2}\right]} \Gamma\left(a-k+\frac{1}{2}+\left[\frac{n+1}{2}\right]\right) & \\
& \times(-i s)^{\frac{1-(-1)^{n}}{2}}{ }_{1} F_{1}\left(\begin{array}{c|c}
\frac{1}{2}+a-k+\left[\frac{n+1}{2}\right] & -\frac{s^{2}}{2} \\
1-\frac{(-1)^{n}}{2} & .
\end{array}\right.
\end{aligned}
$$

The second way to compute $I_{n, k}(s ; a)$ is that we respectively consider $n=2 m$ and $n=2 m+1$ and directly apply the cosine and sine Fourier transforms of the function $e^{-\frac{x^{2}}{2}} x^{2 a+n-2 k}$; see [7, Section 1.4, formula 14] and [7, Section 2.4, formula $24]$. In other words, by noting that $(-1)^{2 a}=1$ we have

$$
\begin{aligned}
I_{2 m, k}(s ; a) & =\int_{-\infty}^{\infty} \cos (s x) x^{2 a+2 m-2 k} e^{-\frac{1}{2} x^{2}} \mathrm{~d} x-i \int_{-\infty}^{\infty} \sin (s x) x^{2 a+2 m-2 k} e^{-\frac{1}{2} x^{2}} \mathrm{~d} x \\
& =2 \int_{0}^{\infty} \cos (s x) x^{2 a+2 m-2 k} e^{-\frac{1}{2} x^{2}} \mathrm{~d} x \\
& =2^{a+m-k+\frac{1}{2}} \Gamma\left(a+m-k+\frac{1}{2}\right){ }_{1} F_{1}\left(\begin{array}{c}
a+m-k+\frac{1}{2} \\
\frac{1}{2}
\end{array} \mid-\frac{s^{2}}{2}\right)
\end{aligned}
$$


and

$$
\begin{aligned}
I_{2 m,+1 k}(s ; a)= & \int_{-\infty}^{\infty} \cos (s x) x^{2 a+2 m+1-2 k} e^{-\frac{1}{2} x^{2}} \mathrm{~d} x \\
& -i \int_{-\infty}^{\infty} \sin (s x) x^{2 a+2 m+1-2 k} e^{-\frac{1}{2} x^{2}} \mathrm{~d} x \\
= & -2 i \int_{0}^{\infty} \sin (s x) x^{2 a+2 m+1-2 k} e^{-\frac{1}{2} x^{2}} \mathrm{~d} x \\
= & (-i s) 2^{a+m-k+\frac{3}{2}} \Gamma\left(a+m-k+\frac{3}{2}\right)_{1} F_{1}\left(\begin{array}{c}
a+m-k+\frac{3}{2} \\
\frac{3}{2}
\end{array} \mid-\frac{s^{2}}{2}\right) .
\end{aligned}
$$

Consequently, the result (2.29) simplifies (2.23) as

$$
\begin{aligned}
\mathbf{F}(u)(s)=\Gamma & \left(a+\frac{1}{2}+\left[\frac{n+1}{2}\right]\right) 2^{a+\frac{1}{2}+\left[\frac{n+1}{2}\right]} \\
& \times(-i s)^{\frac{1-(-1)^{n}}{2}} \sum_{k=0}^{\left[\frac{n}{2}\right]} \frac{\left(-\left[\frac{n}{2}\right]\right)_{k}\left(\frac{1}{2}-b-\left[\frac{n+1}{2}\right]\right)_{k}}{\left(\frac{1}{2}-a-\left[\frac{n+1}{2}\right]\right)_{k} 2^{k} k !} \\
& \times{ }_{1} F_{1}\left(\begin{array}{c|c}
\frac{1}{2}+a-k+\left[\frac{n+1}{2}\right] & \left.-\frac{s^{2}}{2}\right) . \\
1-\frac{(-1)^{n}}{2}
\end{array}\right.
\end{aligned}
$$

On the other hand, according to Kummer's formula [1],

$$
{ }_{1} F_{1}\left(\begin{array}{c|c}
a & x \\
c & x
\end{array}\right)=e_{1}^{x}{ }_{1} F_{1}\left(\begin{array}{c|c}
c-a & -x \\
c &
\end{array}\right)
$$

relation (2.32) can be transformed to

$$
\begin{aligned}
& \mathbf{F}(u)(s)=\Gamma\left(a+\frac{1}{2}+\left[\frac{n+1}{2}\right]\right) 2^{a+\frac{1}{2}+\left[\frac{n+1}{2}\right]} e^{-\frac{1}{2} x^{2}} \\
& \times(-i s)^{\frac{1-(-1)^{n}}{2}} \sum_{k=0}^{\left[\frac{n}{2}\right]} \frac{\left(-\left[\frac{n}{2}\right]\right)_{k}\left(\frac{1}{2}-b-\left[\frac{n+1}{2}\right]\right)_{k}}{\left(\frac{1}{2}-a-\left[\frac{n+1}{2}\right]\right)_{k} 2^{k} k !} \\
& \times{ }_{1} F_{1}\left(\begin{array}{c|c}
-a+k-\left[\frac{n}{2}\right] & 1 \\
1-\frac{(-1)^{n}}{2} & 2
\end{array} s^{2}\right) \text {. }
\end{aligned}
$$

Now, by noting (2.34), if for simplicity we define

$$
\begin{aligned}
J_{n}\left(x ; q_{1}, q_{2}\right)=(x)^{\frac{1-(-1)^{n}}{2}} \sum_{k=0}^{\left[\frac{n}{2}\right]} \frac{\left(-\left[\frac{n}{2}\right]\right)_{k}\left(\frac{1}{2}-q_{2}-\left[\frac{n+1}{2}\right]\right)_{k}}{\left(\frac{1}{2}-q_{1}-\left[\frac{n+1}{2}\right]\right)_{k} 2^{k} k !} \\
\quad \times{ }_{1} F_{1}\left(\begin{array}{c|c}
-q_{1}+k-\left[\frac{n}{2}\right] & 1 \\
1-\frac{(-1)^{n}}{2} & \frac{1}{2} s^{2}
\end{array}\right),
\end{aligned}
$$


then by referring to definitions (2.22) and applying Parseval's identity we get

$$
\begin{aligned}
2 \pi \int_{-\infty}^{\infty} x^{2(a+c)} e^{-x^{2}} H_{n}^{(b)}(x) H_{m}^{(d)}(x) \mathrm{d} x \\
=i^{\frac{(-1)^{n}-(-1)^{m}}{2}} \frac{\Gamma\left(a+\frac{1}{2}+\left[\frac{n+1}{2}\right]\right) \Gamma\left(c+\frac{1}{2}+\left[\frac{m+1}{2}\right]\right)}{2^{-\left(a+\frac{1}{2}+\left[\frac{n+1}{2}\right]\right)} 2^{-\left(c+\frac{1}{2}+\left[\frac{m+1}{2}\right]\right)}} \\
\quad \times \int_{-\infty}^{\infty} e^{-s^{2}} J_{n}(s ; a, b) J_{m}(s ; c, d) \mathrm{d} s .
\end{aligned}
$$

Finally it is sufficient to assume in (2.36) that $b=d=a+c$ and then refer to the orthogonality relation (1.11) to reach the following theorem.

Theorem 2.5. The special function defined in (2.35) satisfies the orthogonality relation

$\int_{-\infty}^{\infty} e^{-x^{2}} J_{n}(x ; a, b) J_{m}(x ; b-a, b) \mathrm{d} x=\frac{\pi 2^{-b-2\left[\frac{n+1}{2}\right]} \Gamma\left(\left[\frac{n}{2}\right]+1\right) \Gamma\left(b+\frac{1}{2}+\left[\frac{n+1}{2}\right]\right)}{\Gamma\left(a+\frac{1}{2}+\left[\frac{n+1}{2}\right]\right) \Gamma\left(b-a+\frac{1}{2}+\left[\frac{n+1}{2}\right]\right)} \delta_{n, m}$

where $a, b>-\frac{1}{2}$ and $(-1)^{2 b}=1$.

\section{REFERENCES}

1. W. Al-Salam, W. R. Allaway and R. Askey, Sieved ultraspherical polynomials, Trans. Amer. Math. Soc., 284 (1984) 39-55. MR.742411 (85j:33005)

2. R. Askey, An integral of Ramanujan and orthogonal polynomials, J. Indian Math. Soc. 51 (1987) 27-36. MR988306 (90d:33004)

3. R. Askey, Orthogonal polynomials old and new, and some combinatorial connections, Enumeration and Design, (D.M. Jacson and S.A. Vanstone, eds.), Academic Press, New York, 1984, 67-84. MR782309 (87a:05022)

4. W. N. Bailey, Generalized Hypergeometric Series. Cambridge Tracts 32, Cambridge University PFTV, 1935. Reprinted by Hafner Publishing Company, 1972. MR0185155 (32:2625)

5. T. S. Chihara, Introduction to Orthogonal Polynomials, Gordon \& Breach, New York, 1978. MR0481884 (58:1979)

6. Digital Library of Mathematical Functions, DLMF available at http://dlmf.nist.gov/

7. A. Erdélyi, W. Magnus, F. Oberhettinger and F. G. Tricomi, Tables of Integral Transforms. Vol. 2, McGraw-Hill, 1954. MR0065685 (16:468c)

8. M. E. H. Ismail, On sieved orthogonal polynomials III: Orthogonality on several intervals, Trans. Amer. Math. Soc., 294 (1986) 89-111. MR819937 (87j:33014b)

9. M. E. H. Ismail, Classical and Quantum Orthogonal Polynomials in One Variable, Encycl. of Math. 98, Cambridge Univ. Press, Cambridge, 2005. MR2191786 (2007f:33001)

10. H. T. Koelink, On Jacobi and continuous Hahn polynomials, Proc. Amer. Math. Soc., 124 (1996) 887-898. MR1307541 (96f:33018)

11. W. Koepf, Hypergeometric Summation. Braunschweig/Wiesbaden, Vieweg, 1988. MR.1644447 (2000c:33002)

12. W. Koepf and M. Masjed-Jamei, Two classes of special functions using Fourier transforms of some finite classes of classical orthogonal polynomials, Proc. Amer. Math. Soc., 135 (2007) 3599-3606. MR2336575 (2008j:33004)

13. T. H. Koornwinder, Special orthogonal polynomial systems mapped onto each other by the Fourier-Jacobi transform, Polynômes Orthogonaux et Applications (C. Brezinski, A. Draux, A. P. Magnus, P. Maroni and A. Ronveaux, Eds.), Lecture Notes in Math. 1171, Springer, 1985, 174-183. MR838982 (87g:33007)

14. M. Masjed-Jamei, A basic class of symmetric orthogonal polynomials using the extended Sturm-Liouville theorem for symmetric functions, J. Math. Anal. Appl., 325 (2007) 753-775. MR2270049 (2008c:33008) 
15. M. Masjed-Jamei, Biorthogonal exponential sequences with weight function on the real line and an orthogonal sequence of trigonometric functions, Proc. Amer. Math. Soc., 136 (2008) 409-417. MR2358478 (2009a:42040)

16. L. J. Rogers, Third memoir on the expansion of certain infinite products, Proc. London Math. Soc. 26 (1895) 15-32.

17. G. Szegő, Orthogonal polynomials, American Mathematical Society Colloquium Publications, Vol. 23, Providence, RI, 1975. MR0372517 (51:8724)

18. E. T. Whittaker and G. N. Watson, A Course of Modern Analysis, 4th ed., Cambridge University Press, Cambridge, 1927. MR:1424469 (97k:01072)

Department of Mathematics, K. N. Toosi University of Technology, P.O. Box 163151618, Tehran, Iran - And - School of Mathematics, Institute for Research in Fundamental Sciences (IPM), P. O. Box 19395-5746, Tehran, Iran

E-mail address: mmjamei@kntu.ac.ir, mmjamei@yahoo.com

Institute of Mathematics, University of Kassel, Heinrich-Plett-Str. 40, D-34132 Kassel, Germany

E-mail address: koepf@mathematik.uni-kassel.de 